\title{
Positive Solutions of Singular Complementary Lidstone Boundary Value Problems
}

\author{
Ravi P. Agarwal, ${ }^{1}$ Donal O'Regan, ${ }^{2}$ and Svatoslav Staněk ${ }^{3}$ \\ ${ }^{1}$ Department of Mathematical Sciences, Florida Institute of Technology, Melbourne, \\ FL 32901-6975, USA \\ ${ }^{2}$ Department of Mathematics, National University of Ireland, Galway, Ireland \\ ${ }^{3}$ Department of Mathematical Analysis, Faculty of Science, Palacký University, Tř. 17. listopadu 12, \\ 77146 Olomouc, Czech Republic
}

Correspondence should be addressed to Ravi P. Agarwal, agarwal@fit.edu

Received 7 October 2010; Accepted 21 November 2010

Academic Editor: Irena Rachůnková

Copyright (C) 2010 Ravi P. Agarwal et al. This is an open access article distributed under the Creative Commons Attribution License, which permits unrestricted use, distribution, and reproduction in any medium, provided the original work is properly cited.

We investigate the existence of positive solutions of singular problem $(-1)^{m} x^{(2 m+1)}=f(t, x, \ldots$, $\left.x^{(2 m)}\right), x(0)=0, x^{(2 i-1)}(0)=x^{(2 i-1)}(T)=0,1 \leq i \leq m$. Here, $m \geq 1$ and the Carathéodory function $f\left(t, x_{0}, \ldots, x_{2 m}\right)$ may be singular in all its space variables $x_{0}, \ldots, x_{2 m}$. The results are proved by regularization and sequential techniques. In limit processes, the Vitali convergence theorem is used.

\section{Introduction}

Let $T$ be a positive constant, $J=[0, T]$ and $\mathbb{R}_{-}=(-\infty, 0), \mathbb{R}_{+}=(0, \infty), \mathbb{R}_{0}=\mathbb{R} \backslash\{0\}$. We consider the singular complementary Lidstone boundary value problem

$$
\begin{aligned}
& (-1)^{m} x^{(2 m+1)}(t)=f\left(t, x(t), \ldots, x^{(2 m)}(t)\right), \quad m \geq 1, \\
& x(0)=0, \quad x^{(2 i-1)}(0)=x^{(2 i-1)}(T)=0, \quad 1 \leq i \leq m,
\end{aligned}
$$

where $f$ satisfies the local Carathéodory function on $J \times \Phi(f \in \operatorname{Car}(J \times \Phi))$ with

$$
\boldsymbol{\Phi}= \begin{cases}\underbrace{\mathbb{R}_{+}^{2} \times \mathbb{R}_{0} \times \mathbb{R} \times \mathbb{R}_{0} \times \mathbb{R}_{+} \times \cdots \times \mathbb{R}_{+} \times \mathbb{R}_{0}}_{4 k-1} & \text { if } m=2 k-1, \\ \underbrace{\mathbb{R}_{+}^{2} \times \mathbb{R}_{0} \times \mathbb{R} \times \mathbb{R}_{0} \times \mathbb{R}_{+} \times \cdots \times \mathbb{R}_{-} \times \mathbb{R}_{0}}_{4 k+1} & \text { if } m=2 k .\end{cases}
$$


The function $f\left(t, x_{0}, \ldots, x_{2 m}\right)$ is positive and may be singular at the value zero of all its space variables $x_{0}, \ldots, x_{2 m}$.

Let $i \in\{0,1, \ldots, 2 m\}$. We say that $f$ is singular at the value zero of its space variable $x_{i}$ if for a.e. $t \in J$ and all $x_{j}, 0 \leq j \leq 2 m, j \neq i$ such that $\left(x_{0}, \ldots, x_{i}, \ldots, x_{2 m}\right) \in \mathscr{\Phi}$, the relation

$$
\lim _{x_{i} \rightarrow 0} f\left(t, x_{0}, \ldots, x_{i}, \ldots, x_{2 m}\right)=\infty
$$

holds.

A function $x \in A C^{2 m}(J)$ (i.e., $x$ has absolutely continuous $2 m$ th derivative on $J$ ) is a positive solution of problem (1.1), (1.2) if $x(t)>0$ for $t \in(0, T], x$ satisfies the boundary conditions (1.2) and (1.1) holds a.e. on $J$.

The regular complementary Lidstone problem

$$
\begin{gathered}
(-1)^{m} x^{(2 m+1)}(t)=h\left(t, x(t), \ldots, x^{(q)}(t)\right), \quad m \geq 1, q \text { fixed, } 0 \leq q \leq 2 m, \\
x(0)=\alpha_{0}, \quad x^{(2 i-1)}(0)=\alpha_{i}, \quad x^{(2 i-1)}(1)=\beta_{i}, \quad 1 \leq i \leq m
\end{gathered}
$$

was discussed in [1]. Here, $h:[0,1] \times \mathbb{R}^{q+1} \rightarrow \mathbb{R}$ is continuous at least in the interior of the domain of interest. Existence and uniqueness criteria for problem (1.5) are proved by the complementary Lidstone interpolating polynomial of degree $2 m$. No contributions exist, as far as we know, concerning the existence of positive solutions of singular complementary Lidstone problems.

We observe that differential equations in complementary Lidstone problems as well as derivatives in boundary conditions are odd orders, in contrast to the Lidstone problem

$$
\begin{gathered}
(-1)^{m} x^{(2 m)}(t)=p\left(t, x(t), \ldots, x^{(r)}(t)\right), \quad m \geq 1, r \text { fixed, } 0 \leq r \leq 2 m-1, \\
x^{(2 i)}(0)=a_{i}, \quad x^{(2 i)}(1)=b_{i}, \quad 1 \leq i \leq m-1
\end{gathered}
$$

where the differential equation and derivatives in the boundary conditions are even orders. For $a_{i}=b_{i}=0(1 \leq i \leq m-1)$, regular Lidstone problems were discussed in [2-9], while singular ones in [10-15].

The aim of this paper is to give the conditions on the function $f$ in (1.1) which guarantee that the singular problem (1.1), (1.2) has a solution. The existence results are proved by regularization and sequential techniques, and in limit processes, the Vitali convergence theorem $[16,17]$ is applied.

Throughout the paper, $\|x\|_{\infty}=\max \{|x(t)|: t \in J\}$ and $\|x\|_{C^{n}}=\sum_{k=0}^{n}\left\|x^{(k)}\right\|_{\infty}, n \geq 1$ stands for the norm in $C^{0}(J)$ and $C^{n}(J)$, respectively. $L^{1}(J)$ denotes the set of functions (Lebesgue) integrable on $J$ and meas $\mathcal{M}$ the Lebesgue measure of $\mathcal{M} \subset J$.

We work with the following conditions on the function $f$ in (1.1).

$\left(H_{1}\right) f \in \operatorname{Car}(J \times \mathscr{\nexists})$ and there exists $a \in(0, \infty)$ such that

$$
a \leq f\left(t, x_{0}, \ldots, x_{2 m}\right)
$$

for a.e. $t \in J$ and each $\left(x_{0}, \ldots, x_{2 m}\right) \in \boldsymbol{\Phi}$. 
$\left(H_{2}\right)$ For a.e. $t \in J$ and for all $\left(x_{0}, \ldots, x_{2 m}\right) \in \Phi$, the inequality

$$
f\left(t, x_{0}, \ldots, x_{2 m}\right) \leq h\left(t, \sum_{j=0}^{2 m}\left|x_{j}\right|\right)+\sum_{j=0}^{2 m} \omega_{j}\left(\left|x_{j}\right|\right)
$$

is fulfilled, where $h \in \operatorname{Car}(J \times[0, \infty))$ is positive and nondecreasing in the second variable, $\omega_{j}: \mathbb{R}_{+} \rightarrow \mathbb{R}_{+}$is nonincreasing, $0 \leq j \leq 2 m$,

$$
\begin{gathered}
\limsup _{v \rightarrow \infty} \frac{1}{v} \int_{0}^{T} h(t, K v) \mathrm{d} t<1, \quad K= \begin{cases}\frac{T^{2 m+1}-1}{T-1} & \text { if } T \neq 1, \\
2 m+1 & \text { if } T=1,\end{cases} \\
\int_{0}^{1} \omega_{2 j}\left(s^{2}\right) \mathrm{d} s<\infty, \quad \int_{0}^{1} \omega_{2 j+1}(s) \mathrm{d} s<\infty \quad \text { if } 0 \leq j \leq m-1, \\
\int_{0}^{1} \omega_{2 m}(s) \mathrm{d} s<\infty .
\end{gathered}
$$

The paper is organized as follows. In Section 2, we construct a sequence of auxiliary regular differential equations associated with (1.1). Section 3 is devoted to the study of auxiliary regular complementary Lidstone problems. We show that the solvability of these problems is reduced to the existence of a fixed point of an operator $\mathcal{H}$. The existence of a fixed point of $\mathscr{H}$ is proved by a fixed point theorem of cone compression type according to GuoKrasnosel'skii $[18,19]$. The properties of solutions to auxiliary problems are also investigated here. In Section 4, applying the results of Section 3, the existence of a positive solution of the singular problem (1.1), (1.2) is proved.

\section{Regularization}

Let $m$ be from (1.1). For $n \in \mathbb{N}$, define $\chi_{n}, \varphi_{n}, \tau_{n, m} \in C^{0}(\mathbb{R}), \mathbb{R}_{n} \subset \mathbb{R}$, and $\Phi_{n} \subset \mathbb{R}^{2 m+1}$ by the formulas

$$
\begin{gathered}
x_{n}(u)=\left\{\begin{array}{ll}
u & \text { for } u \geq \frac{1}{n}, \\
\frac{1}{n} & \text { for } u<\frac{1}{n},
\end{array} \quad \varphi_{n}(u)= \begin{cases}-\frac{1}{n} & \text { for } u>-\frac{1}{n}, \\
u & \text { for } u \leq-\frac{1}{n},\end{cases} \right. \\
\tau_{n, m}=\left\{\begin{array}{ll}
x_{n} & \text { if } m=2 k-1, \\
\varphi_{n} & \text { if } m=2 k,
\end{array} \mathbb{R}_{n}=\left(-\infty,-\frac{1}{n}\right] \cup\left[\frac{1}{n}, \infty\right),\right. \\
\Phi_{n}=\underbrace{\mathbb{R}^{2} \times \mathbb{R}_{n} \times \mathbb{R} \times \mathbb{R}_{n} \times \mathbb{R} \times \cdots \times \mathbb{R} \times \mathbb{R}_{n}}_{2 m+1} .
\end{gathered}
$$


Let $f \in \operatorname{Car}(J \times \Phi)$. Chose $n \in \mathbb{N}$ and put

$$
\begin{aligned}
& f_{n}^{*}\left(t, x_{0}, x_{1}, x_{2}, x_{3}, x_{4}, \ldots, x_{2 m-1}, x_{2 m}\right) \\
& \quad=f\left(t, x_{n}\left(x_{0}\right), x_{n}\left(x_{1}\right), x_{2}, \varphi_{n}\left(x_{3}\right), x_{4}, \ldots, \tau_{n, m}\left(x_{2 m-1}\right), x_{2 m}\right)
\end{aligned}
$$

for $\left(t, x_{0}, x_{1}, x_{2}, x_{3}, x_{4}, \ldots, x_{2 m-1}, x_{2 m}\right) \in J \times \Phi_{n}$. Now, define an auxiliary function $f_{n}$ by means of the following recurrence formulas:

$$
\begin{aligned}
& f_{n, 0}\left(t, x_{0}, x_{1}, \ldots, x_{2 m}\right)=f_{n}^{*}\left(t, x_{0}, x_{1}, \ldots, x_{2 m}\right) \text { for }\left(t, x_{0}, x_{1}, \ldots, x_{2 m}\right) \in J \times \Phi_{n}, \\
& f_{n, i}\left(t, x_{0}, x_{1}, \ldots, x_{2 m}\right) \\
& = \begin{cases}f_{n, i-1}\left(t, x_{0}, x_{1}, \ldots, x_{2 m}\right) & \text { if }\left|x_{2 i}\right| \geq \frac{1}{n^{\prime}} \\
\frac{n}{2}\left[f_{n, i-1}\left(t, x_{0}, \ldots, x_{2 i-1}, \frac{1}{n}, x_{2 i+1}, \ldots, x_{2 m}\right)\left(x_{2 i}+\frac{1}{n}\right)\right. & \\
\left.\quad-f_{n, i-1}\left(t, x_{0}, \ldots, x_{2 i-1},-\frac{1}{n}, x_{2 i+1}, \ldots, x_{2 m}\right)\left(x_{2 i}-\frac{1}{n}\right)\right] & \text { if }\left|x_{2 i}\right|<\frac{1}{n}\end{cases}
\end{aligned}
$$

for $1 \leq i \leq m$, and

$$
f_{n}\left(t, x_{0}, x_{1}, \ldots, x_{2 m}\right)=f_{n, m}\left(t, x_{0}, x_{1}, \ldots, x_{2 m}\right) \quad \text { for }\left(t, x_{0}, x_{1}, \ldots, x_{2 m}\right) \in J \times \mathbb{R}^{2 m+1} .
$$

Then, under condition $\left(H_{1}\right), f_{n} \in \operatorname{Car}\left(J \times \mathbb{R}^{2 m+1}\right)$ and

$$
a \leq f_{n}\left(t, x_{0}, x_{1}, \ldots, x_{2 m}\right) \text { for a.e. } t \in J \text { and } \operatorname{all}\left(x_{0}, x_{1}, \ldots, x_{2 m}\right) \in \mathbb{R}^{2 m+1} .
$$

Condition $\left(\mathrm{H}_{2}\right)$ gives

$$
\begin{gathered}
f_{n}\left(t, x_{0}, x_{1}, \ldots, x_{2 m}\right) \leq h\left(t, 2 m+1+\sum_{j=0}^{2 m}\left|x_{j}\right|\right)+\sum_{j=0}^{2 m}\left(\omega_{j}\left(\left|x_{j}\right|\right)+\omega_{j}(1)\right), \\
\text { for a.e. } t \in J \text { and all }\left(x_{0}, x_{1}, \ldots, x_{2 m}\right) \in \mathbb{R}_{0}^{2 m+1}, \\
f_{n}\left(t, x_{0}, x_{1}, \ldots, x_{2 m}\right) \leq h\left(t, 2 m+1+\sum_{j=0}^{2 m}\left|x_{j}\right|\right)+\sum_{j=0}^{2 m} \omega_{j}\left(\frac{1}{n}\right) \\
\text { for a.e. } t \in J \text { and all }\left(x_{0}, x_{1}, \ldots, x_{2 m}\right) \in \mathbb{R}^{2 m+1} .
\end{gathered}
$$

We investigate the regular differential equation

$$
(-1)^{m} x^{(2 m+1)}(t)=f_{n}\left(t, x(t), \ldots, x^{(2 m)}(t)\right) .
$$

If a function $x \in A C^{2 m}(J)$ satisfies (2.8) for a.e. $t \in J$, then $x$ is called a solution of (2.8). 


\section{Auxiliary Regular Problems}

Let $j \in \mathbb{N}$ and denote by $G_{j}(t, s)$ the Green function of the problem

$$
x^{(2 j)}(t)=0, \quad x^{(2 i)}(0)=x^{(2 i)}(T)=0, \quad 0 \leq i \leq j-1 .
$$

Then,

$$
G_{1}(t, s)= \begin{cases}\frac{s}{T}(t-T) & \text { for } 0 \leq s \leq t \leq T \\ \frac{t}{T}(s-T) & \text { for } 0 \leq t \leq s \leq T\end{cases}
$$

By $[2,3,20]$, the Green function $G_{j}$ can be expressed as

$$
G_{j}(t, s)=\int_{0}^{T} G_{1}(t, \tau) G_{j-1}(\tau, s) \mathrm{d} \tau, \quad j>1,
$$

and it is known that (see, e.g., $[3,20])$

$$
(-1)^{j} G_{j}(t, s)>0 \quad \text { for }(t, s) \in(0, T) \times(0, T), j \geq 1 \text {. }
$$

Lemma 3.1 (see [10, Lemmas 2.1 and 2.3]). For $(t, s) \in J \times J$ and $j \in \mathbb{N}$, the inequalities

$$
\begin{gathered}
(-1)^{j} G_{j}(t, s) \leq \frac{T^{2 j-3}}{6^{j-1}} s(T-s), \\
(-1)^{j} G_{j}(t, s) \geq \frac{T^{2 j-5}}{30^{j-1}} t s(T-t)(T-s)
\end{gathered}
$$

hold.

Let $\gamma \in L^{1}(J)$ and let $u \in A C^{2 m-1}(J)$ be a solution of the differential equation

$$
(-1)^{m} u^{(2 m)}(t)=\gamma(t)
$$

satisfying the Lidstone boundary conditions

$$
u^{(2 i)}(0)=u^{(2 i)}(T)=0, \quad 0 \leq i \leq m-1 .
$$

It follows from the definition of the Green function $G_{j}$ that

$$
(-1)^{j} u^{(2 j)}(t)=(-1)^{m-j} \int_{0}^{T} G_{m-j}(t, s) \gamma(s) \mathrm{d} s \quad \text { for } t \in J, 0 \leq j \leq m-1 .
$$


It is easy to check that $x \in A C^{2 m}(J)$ is a solution of problem (2.8), (1.2) if and only if $x(0)=0$, and its derivative $x^{\prime}$ is a solution of a problem involving the functional differential equation

$$
(-1)^{m} u^{(2 m)}(t)=f_{n}\left(t, \int_{0}^{t} u(s) \mathrm{d} s, u(t), \ldots, u^{(2 m-1)}(t)\right)
$$

and the Lidstone boundary conditions (3.8). From (3.9) (for $j=0$ ), we see that $u \in A C^{2 m-1}(J)$ is a solution of problem (3.10), (3.8) exactly if it is a solution of the equation

$$
u(t)=(-1)^{m} \int_{0}^{T} G_{m}(t, s) f_{n}\left(s, \int_{0}^{s} u(\tau) \mathrm{d} \tau, u(s), \ldots, u^{(2 m-1)}(s)\right) \mathrm{d} s,
$$

in the set $C^{2 m-1}(J)$. Consequently, $x$ is a solution of problem (2.8), (1.2) if and only if it is a solution of the equation

$$
x(t)=(-1)^{m} \int_{0}^{t}\left(\int_{0}^{T} G_{m}(s, \tau) f_{n}\left(\tau, x\left(\tau, \ldots, x^{(2 m)}(\tau)\right)\right) \mathrm{d} \tau\right) \mathrm{d} s,
$$

in the set $C^{2 m}(J)$. It means that $x$ is a solution of problem $(2.8),(1.2)$ if $x$ is a fixed point of the operator $\mathscr{d e}: C^{2 m}(J) \rightarrow C^{2 m}(J)$ defined as

$$
(\mathscr{L} x)(t)=(-1)^{m} \int_{0}^{t}\left(\int_{0}^{T} G_{m}(s, \tau) f_{n}\left(\tau, x\left(\tau, \ldots, x^{(2 m)}(\tau)\right)\right) \mathrm{d} \tau\right) \mathrm{d} s
$$

We prove the existence of a fixed point of $\mathscr{t}$ by the following fixed point result of cone compression type according to Guo-Krasnosel'skii (see, e.g., $[18,19])$.

Lemma 3.2. Let $X$ be a Banach space, and let $P \subset X$ be a cone in $X$. Let $\Omega_{1}, \Omega_{2}$ be bounded open balls of X centered at the origin with $\bar{\Omega}_{1} \subset \Omega_{2}$. Suppose that $\mathcal{F}: P \cap\left(\bar{\Omega}_{2} \backslash \Omega_{1}\right) \rightarrow P$ is completely continuous operator such that

$$
\|\mp x\| \geq\|x\| \quad \text { for } x \in P \cap \partial \Omega_{1}, \quad\|\mp x\| \leq\|x\| \quad \text { for } x \in P \cap \partial \Omega_{2}
$$

holds. Then, $\mathcal{F}$ has a fixed point in $P \cap\left(\bar{\Omega}_{2} \backslash \Omega_{1}\right)$.

We are now in the position to prove that problem (2.8), (1.2) has a solution.

Lemma 3.3. Let $\left(H_{1}\right)$ and $\left(H_{2}\right)$ hold. Then, problem (2.8), (1.2) has a solution.

Proof. Let the operator $\mathscr{H}: C^{2 m}(J) \rightarrow C^{2 m}(J)$ be given in (3.13), and let

$$
P=\left\{x \in C^{2 m}(J): x(t) \geq 0 \text { for } t \in J\right\}
$$

Then, $P$ is a cone in $C^{2 m}(J)$ and since $(-1)^{m} G_{m}(t, s)>0$ for $(t, s) \in(0, T) \times(0, T)$ by $(3.4)$ and $f_{n}$ satisfies (2.5), we see that $\mathscr{H}: C^{2 m}(J) \rightarrow P$. The fact that $\mathscr{L}$ is a completely continuous 
operator follows from $f_{n} \in \operatorname{Car}\left(J \times \mathbb{R}^{2 m+1}\right)$, from Lebesgue dominated convergence theorem, and from the Arzelà-Ascoli theorem.

Choose $x \in P$ and put $y(t)=(\mathscr{\ell} x)(t)$ for $t \in J$. Then, (cf. (2.5))

$$
(-1)^{m} y^{(2 m+1)}(t)=f_{n}\left(t, x(t), \ldots, x^{(2 m)}(t)\right) \geq a>0 \quad \text { for a.e. } t \in J .
$$

Since $y(0)=0$ and $y^{(2 i-1)}(0)=y^{(2 i-1)}(T)=0$ for $1 \leq i \leq m$, the equality $y^{(j)}\left(\xi_{j}\right)=0$ holds with some $\xi_{j} \in J$ for $0 \leq j \leq 2 m$. We now use the equality $y^{(2 m)}\left(\xi_{2 m}\right)=0$ and have

$$
\left|y^{(2 m)}(t)\right|=\left|\int_{\xi_{2 m}}^{t} y^{(2 m+1)}(s) \mathrm{d} s\right| \geq a\left|t-\xi_{2 m}\right| \quad \text { for } t \in J .
$$

Hence, $\left\|y^{(2 m)}\right\|_{\infty} \geq a T / 2$, and so

$$
\|\mathscr{H} x\|_{C^{2 m}}>\frac{a T}{2}
$$

Next, we deduce from the relation

$$
\left|y^{(2 m)}(t)\right|=\left|\int_{\xi_{2 m}}^{t} f_{n}\left(s, x(s), \ldots, x^{(2 m)}(s)\right) \mathrm{d} s\right| \leq \int_{0}^{T} f_{n}\left(s, x(s), \ldots, x^{(2 m)}(s)\right) \mathrm{d} s
$$

and from (2.7) that

$$
\left|y^{(2 m)}(t)\right| \leq \int_{0}^{T} h\left(s, 2 m+1+\|x\|_{C^{2 m}}\right) \mathrm{d} s+T \sum_{j=0}^{2 m} \omega_{j}\left(\frac{1}{n}\right) \quad \text { for } t \in J .
$$

Therefore,

$$
\left\|y^{(2 m)}\right\|_{\infty} \leq \int_{0}^{T} h\left(s, 2 m+1+\|x\|_{C^{2 m}}\right) \mathrm{d} s+V
$$

where $V=T \sum_{j=0}^{2 m} \omega_{j}(1 / n)$. Since $y^{(j)}\left(\xi_{j}\right)=0$ for $0 \leq j \leq 2 m$, we have

$$
\left\|y^{(j)}\right\|_{\infty} \leq T^{2 m-j}\left\|y^{(2 m)}\right\|_{\infty}, \quad 0 \leq j \leq 2 m
$$

The last inequality together with (3.21) gives

$$
\|y\|_{C^{2 m}} \leq K\left\|y^{(2 m)}\right\|_{\infty} \leq K\left(\int_{0}^{T} h\left(s, 2 m+1+\|x\|_{C^{2 m}}\right) \mathrm{d} s+V\right),
$$

where $K$ is from $\left(H_{2}\right)$. Since $x \in P$ is arbitrary, relations (3.18) and (3.21) imply that for all 
$x \in P$, inequalities (3.18) and

$$
\|\mathscr{H} x\|_{C^{2 m}} \leq K\left(\int_{0}^{T} h\left(s, 2 m+1+\|x\|_{C^{2 m}}\right) \mathrm{d} s+V\right)
$$

hold. By $\left(H_{2}\right)$, there exists $C>0$ such that

$$
\frac{1}{v}\left(\int_{0}^{T} h(s, 2 m+1+K v) \mathrm{d} s+V\right) \leq 1 \quad \forall v \geq \frac{C}{K^{\prime}}
$$

and therefore,

$$
K\left(\int_{0}^{T} h(s, 2 m+1+v) \mathrm{d} s+V\right) \leq v \quad \forall v \geq C .
$$

Let

$$
\Omega_{1}=\left\{x \in C^{2 m}(J):\|x\|_{C^{2 m}}<\frac{a T}{2}\right\}, \quad \Omega_{2}=\left\{x \in C^{2 m}(J):\|x\|_{C^{2 m}}<C\right\} .
$$

Then, it follows from (3.18), (3.24), and (3.26) that

$$
\|\mathscr{L} x\|_{C^{2 m}} \geq\|x\|_{C^{2 m}} \quad \text { for } x \in P \cap \partial \Omega_{1}, \quad\|\mathscr{L} x\|_{C^{2 m}} \leq\|x\|_{C^{2 m}} \quad \text { for } x \in P \cap \partial \Omega_{2} .
$$

The conclusion now follows from Lemma $3.2\left(\right.$ for $X=C^{2 m}(J)$ and $\left.\mathscr{F}=\mathscr{\ell}\right)$.

The properties of solutions to problem (2.8), (1.2) are collected in the following lemma.

Lemma 3.4. Let $\left(H_{1}\right)$ and $\left(H_{2}\right)$ be satisfied. Let $x_{n}$ be a solution of problem (2.8), (1.2). Then, for all $n \in \mathbb{N}$, the following assertions hold:

(i) $(-1)^{j} x_{n}^{(2 j+1)}(t)>0$ for $t \in(0, T), 0 \leq j \leq m-1$, and $(-1)^{m} x_{n}^{(2 m+1)}(t) \geq a$ for a.e. $t \in J$,

(ii) $x_{n}$ is increasing on $J$, and for $0 \leq j \leq m-1,(-1)^{j} x_{n}^{(2 j+2)}$ is decreasing on $J$, and there is a unique $\xi_{j, n} \in(0, T)$ such that $x_{n}^{(2 j+2)}\left(\xi_{j, n}\right)=0$,

(iii) there exists a positive constant $A$ such that

$$
\begin{gathered}
\left|x_{n}^{(2 m)}(t)\right| \geq A\left|t-\xi_{m-1, n}\right|, \\
\left|x_{n}^{(2 j+2)}(t)\right| \geq A\left(t-\xi_{j, n}\right)^{2} \quad \text { if } 0 \leq j \leq m-2, \\
\left|x_{n}^{(2 j+1)}(t)\right| \geq A t(T-t) \quad \text { if } 0 \leq j \leq m-1, \\
x_{n}(t) \geq A t^{2},
\end{gathered}
$$

for $t \in J$,

(iv) the sequence $\left\{x_{n}\right\}$ is bounded in $C^{2 m}(J)$. 
Proof. Let us choose an arbitrary $n \in \mathbb{N}$. By (2.5),

$$
(-1)^{m} x_{n}^{(2 m+1)}(t)=f_{n}\left(t, x_{n}(t), \ldots, x_{n}^{(2 m)}(t)\right) \geq a \quad \text { for a.e. } t \in J,
$$

and it follows from the definition of the Green function $G_{j}$ that the equality

$$
(-1)^{j} x_{n}^{(2 j+1)}(t)=(-1)^{m-j} \int_{0}^{T} G_{m-j}(t, s) f_{n}\left(s, x_{n}(s), \ldots, x_{n}^{(2 m)}(s)\right) \mathrm{d} s
$$

holds for $t \in J$ and $0 \leq j \leq m-1$. Now, using (1.2), (3.4), (3.30), and (3.31), we see that assertion (i) is true. Hence, $(-1)^{j} x_{n}^{(2 j+2)}$ is decreasing on $J$ for $0 \leq j \leq m-1$ and $x_{n}$ is increasing on this interval. Due to $x_{n}^{(2 i-1)}(0)=x_{n}^{(2 i-1)}(T)=0$ for $1 \leq i \leq m$, there exists a unique $\xi_{j, n} \in(0, T)$ such that $u_{n}^{(2 j+2)}\left(\xi_{j, n}\right)=0$ for $0 \leq j \leq m-1$. Consequently, assertion (ii) holds.

Next, in view of (2.5), (3.6), and (3.31),

$$
\begin{aligned}
\left|x_{n}^{(2 j+1)}(t)\right| & \geq \frac{T^{2(m-j)-5} a}{30^{m-j-1}} t(T-t) \int_{0}^{T} s(T-s) \mathrm{d} s \\
& =\frac{T^{2(m-j)-2} a}{6 \cdot 30^{m-j-1}} t(T-t) \quad \text { for } t \in J, 0 \leq j \leq m-1 .
\end{aligned}
$$

Since

$$
x_{n}^{(2 j+2)}(t)=\int_{\xi_{j, n}}^{t} x_{n}^{(2 j+3)}(s) \mathrm{d} s
$$

and, by [13, Lemma 6.2],

$$
\left|\int_{\xi_{j, n}}^{t} s(T-s) \mathrm{d} s\right| \geq \frac{T}{6}\left(t-\xi_{j, n}\right)^{2}
$$

we have

$$
\left|x_{n}^{(2 j+2)}(t)\right| \geq \frac{T^{2(m-j)-3} a}{36 \cdot 30^{m-j-2}}\left(t-\xi_{j, n}\right)^{2} \quad \text { for } t \in J, 0 \leq j \leq m-2 .
$$

Furthermore,

$$
\left|x_{n}^{(2 m)}(t)\right|=\left|\int_{\xi_{m-1, n}}^{t} f_{n}\left(s, x_{n}(s), \ldots, x_{n}^{(2 m)}(s)\right) \mathrm{d} s\right| \geq a\left|t-\xi_{m-1, n}\right|, \quad t \in J,
$$


and (cf. (3.32) for $j=0$ )

$$
\begin{aligned}
x_{n}(t) & =\int_{0}^{t} x_{n}^{\prime}(s) \mathrm{d} s \geq \frac{T^{2 m-2} a}{6 \cdot 30^{m-1}} \int_{0}^{t} s(T-s) \mathrm{d} s \\
& =\frac{T^{2 m-2} a}{36 \cdot 30^{m-1}} t^{2}(3 T-2 t) \geq \frac{T^{2 m-1} a}{36 \cdot 30^{m-1}} t^{2} \quad \text { for } t \in J,
\end{aligned}
$$

since $x_{n}^{\prime}>0$ on $(0, T)$ by assertion (ii). Let

$$
A=a \cdot \min \left\{1, A_{1}, A_{2}, \frac{T^{2 m-1}}{36 \cdot 30^{m-1}}\right\},
$$

where

$$
\begin{aligned}
& A_{1}=\min \left\{\frac{T^{2(m-j)-2}}{6 \cdot 30^{m-j-1}}: 0 \leq j \leq m-1\right\}, \\
& A_{2}=\min \left\{\frac{T^{2(m-j)-3}}{36 \cdot 30^{m-j-2}}: 0 \leq j \leq m-2\right\} .
\end{aligned}
$$

Then estimate (3.29) follows from relations (3.32)-(3.37).

It remains to prove the boundedness of the sequence $\left\{x_{n}\right\}$ in $C^{2 m}(J)$. We use estimate (3.29), the properties of $\omega_{j}$ given in $\left(H_{2}\right)$, and the inequality

$$
t(T-t) \geq \begin{cases}\frac{T}{2} t & \text { for } 0<t \leq \frac{T}{2} \\ \frac{T}{2}(T-t) & \text { for } \frac{T}{2}<t<T\end{cases}
$$

and have

$$
\begin{aligned}
\int_{0}^{T} \omega_{2 m}\left(\left|x_{n}^{(2 m)}(s)\right|\right) \mathrm{d} s & \leq \int_{0}^{T} \omega_{2 m}\left(A\left|s-\xi_{m-1, n}\right|\right) \mathrm{d} s \\
& =\frac{1}{A}\left(\int_{0}^{A \xi_{m-1, n}} \omega_{2 m}(s) \mathrm{d} s+\int_{0}^{A\left(T-\xi_{m-1, n}\right)} \omega_{2 m}(s) \mathrm{d} s\right) \\
& <\frac{2}{A} \int_{0}^{A T} \omega_{2 m}(s) \mathrm{d} s \\
\int_{0}^{T} \omega_{2 j+2}\left(\left|x_{n}^{(2 j+2)}(s)\right|\right) \mathrm{d} s & \leq \int_{0}^{T} \omega_{2 j+2}\left(A\left(s-\xi_{j, n}\right)^{2}\right) \mathrm{d} s \\
& =\frac{1}{\sqrt{A}} \int_{-\sqrt{A} \xi_{j, n}}^{\sqrt{A}\left(T-\xi_{j, n}\right)} \omega_{2 j+2}\left(s^{2}\right) \mathrm{d} s
\end{aligned}
$$


Boundary Value Problems

$$
\begin{aligned}
& <\frac{2}{\sqrt{A}} \int_{0}^{\sqrt{A} T} \omega_{2 j+2}\left(s^{2}\right) \mathrm{d} s \quad \text { for } 0 \leq j \leq m-2, \\
\int_{0}^{T} \omega_{2 j+1}\left(\left|x_{n}^{(2 j+1)}(s)\right|\right) \mathrm{d} s & \leq \int_{0}^{T} \omega_{2 j+1}(A s(T-s)) \mathrm{d} s \\
& <\int_{0}^{T / 2} \omega_{2 j+1}\left(\frac{A T s}{2}\right) \mathrm{d} s+\int_{T / 2}^{T} \omega_{2 j+1}\left(\frac{A T(T-s)}{2}\right) \mathrm{d} s \\
& <\frac{4}{A T} \int_{0}^{A T^{2} / 4} \omega_{2 j+1}(s) \mathrm{d} s \quad \text { for } 0 \leq j \leq m-1, \\
\int_{0}^{T} \omega_{0}\left(\left|x_{n}(s)\right|\right) \mathrm{d} s & \leq \int_{0}^{T} \omega_{0}\left(A s^{2}\right) \mathrm{d} s=\frac{1}{\sqrt{A}} \int_{0}^{\sqrt{A} T} \omega_{0}\left(s^{2}\right) \mathrm{d} s .
\end{aligned}
$$

In particular,

$$
\begin{gathered}
\int_{0}^{T} \omega_{2 m}\left(\left|x_{n}^{(2 m)}(s)\right|\right) \mathrm{d} s<\frac{2}{A} \int_{0}^{A T} \omega_{2 m}(s) \mathrm{d} s \\
\int_{0}^{T} \omega_{2 j+2}\left(\left|x_{n}^{(2 j+2)}(s)\right|\right) \mathrm{d} s<\frac{2}{\sqrt{A}} \int_{0}^{\sqrt{A} T} \omega_{2 j+2}\left(s^{2}\right) \mathrm{d} s \quad \text { for } 0 \leq j \leq m-2, \\
\int_{0}^{T} \omega_{2 j+1}\left(\left|x_{n}^{(2 j+1)}(s)\right|\right) \mathrm{d} s<\frac{4}{A T} \int_{0}^{A T^{2} / 4} \omega_{2 j+1}(s) \mathrm{d} s \quad \text { for } 0 \leq j \leq m-1, \\
\int_{0}^{T} \omega_{0}\left(\left|x_{n}(s)\right|\right) \mathrm{d} s \leq \frac{1}{\sqrt{A}} \int_{0}^{\sqrt{A} T} \omega_{0}\left(s^{2}\right) \mathrm{d} s,
\end{gathered}
$$

for all $n \in \mathbb{N}$. Now, from the above estimates, from (2.6) and from $x_{n}^{(2 m)}\left(\xi_{m-1, n}\right)=0$ for some $\xi_{m-1, n} \in(0, T)$, which is proved in (ii), we get

$$
\begin{aligned}
\left|x_{n}^{(2 m)}(t)\right| & =\left|\int_{\xi m-1, n}^{t} f_{n}\left(s, x_{n}(s), \ldots, x_{n}^{(2 m)}(s)\right) \mathrm{d} s\right| \\
& \leq \int_{0}^{T} f_{n}\left(s, x_{n}(s), \ldots, x_{n}^{(2 m)}(s)\right) \mathrm{d} s \\
& \leq \int_{0}^{T} h\left(s, 2 m+1+\sum_{j=0}^{2 m}\left|x_{n}^{(j)}(s)\right|\right) \mathrm{d} s+\sum_{j=0}^{2 m} \int_{0}^{T}\left(\omega_{j}\left(\left|x_{n}^{(j)}(s)\right|\right)+\omega_{j}(1)\right) \mathrm{d} s \\
& <\int_{0}^{T} h\left(s, 2 m+1+\sum_{j=0}^{2 m}\left\|x_{n}^{(j)}\right\|_{\infty}\right) \mathrm{d} s+\Lambda,
\end{aligned}
$$


where

$$
\begin{aligned}
\Lambda= & \frac{2}{A} \int_{0}^{A T} \omega_{2 m}(s) \mathrm{d} s+\frac{2}{\sqrt{A}} \sum_{j=0}^{m-2} \int_{0}^{\sqrt{A} T} \omega_{2 j+2}\left(s^{2}\right) \mathrm{d} s \\
& +\frac{4}{A T} \sum_{j=0}^{m-1} \int_{0}^{A T^{2} / 4} \omega_{2 j+1}(s) \mathrm{d} s+\frac{1}{\sqrt{A}} \int_{0}^{\sqrt{A} T} \omega_{0}\left(s^{2}\right) \mathrm{d} s+T \sum_{j=0}^{2 m} \omega_{j}(1) .
\end{aligned}
$$

Notice that $\Lambda<\infty$ by $\left(H_{2}\right)$. Consequently,

$$
\left\|x_{n}^{(2 m)}\right\|_{\infty}<\int_{0}^{T} h\left(s, 2 m+1+\sum_{j=0}^{2 m}\left\|x_{n}^{(j)}\right\|_{\infty}\right) \mathrm{d} s+\Lambda \quad \text { for } n \in \mathbb{N} .
$$

Since $\left\|x_{n}^{(j)}\right\|_{\infty} \leq T^{2 m-j}\left\|x_{n}^{(2 m)}\right\|_{\infty}$ for $0 \leq j \leq 2 m$, which follows from the fact that $x_{n}^{(j)}$ vanishes in $J$ by (1.2) and assertion (ii), inequality (3.45) yields

$$
\left\|x_{n}^{(2 m)}\right\|_{\infty}<\int_{0}^{T} h\left(s, 2 m+1+K\left\|x_{n}^{(2 \mathrm{~m})}\right\|_{\infty}\right) \mathrm{d} s+\Lambda \quad \text { for } n \in \mathbb{N},
$$

where $K$ is from $\left(\mathrm{H}_{2}\right)$. Due to the condition

$$
\limsup _{v \rightarrow \infty} \frac{1}{v} \int_{0}^{T} h(t, K v) \mathrm{d} v<1
$$

in $\left(H_{2}\right)$, there exists a positive constant $S$ such that for all $v \geq S$ the inequality

$$
\int_{0}^{T} h(t, 2 m+1+K v) \mathrm{d} t+\Lambda \leq v
$$

is fulfilled. The last inequality together with estimate (3.46) gives $\left\|x_{n}^{(2 m)}\right\|_{\infty}<S$ for $n \in \mathbb{N}$. Consequently, $\left\|x_{n}^{(j)}\right\|_{\infty}<T^{2 m-j} S$ for $0 \leq j \leq 2 n, n \in \mathbb{N}$, and assertion (iv) follows.

The following result gives the important property of $\left\{f_{n}\left(t, x_{n}(t), \ldots, x_{n}^{(2 m)}(t)\right)\right\}$ for applying the Vitali convergent theorem in the proof of Theorem 4.1.

Lemma 3.5. Let $\left(H_{1}\right)$ and $\left(H_{2}\right)$ hold. Let $x_{n}$ be a solution of problem (2.8), (1.2). Then, the sequence

$$
\left\{f_{n}\left(t, x_{n}(t), \ldots, x_{n}^{(2 m)}(t)\right)\right\} \subset L^{1}(J)
$$

is uniformly integrable on $J$, that is, for each $\varepsilon>0$, there exists $\delta>0$ such that if $\mathcal{M} \subset J$ and meas $\mathcal{M}<$, then

$$
\int_{\mathcal{M}} f_{n}\left(t, x_{n}(t), \ldots, x_{n}^{(2 m)}(t)\right) \mathrm{d} t<\varepsilon \quad \text { for } n \in \mathbb{N} \text {. }
$$


Proof. By Lemma 3.4 (iv), there exists $S>0$ such that for $n \in \mathbb{N}$, the inequality $\left\|x_{n}\right\|_{C^{2 m}}<S$ holds. Now, we conclude from (2.5) and (2.6), from the properties of $h$ and $\omega_{j}$ given in $\left(H_{2}\right)$, and finally from (3.29) that for $j \in J$ and $n \in \mathbb{N}$, the estimate

$$
\begin{aligned}
a \leq & f_{n}\left(t, x_{n}(t), \ldots, x_{n}^{(2 m)}(t)\right) \\
\leq & h(t, 2 m+1+S)+\omega_{0}\left(A t^{2}\right)+\sum_{j=0}^{m-1} \omega_{2 j+1}(A t(T-t)) \\
& +\sum_{j=0}^{m-2} \omega_{2 j+2}\left(A\left(t-\xi_{j, n}\right)^{2}\right)+\omega_{2 m}\left(A\left|t-\xi_{m-1, n}\right|\right)+\sum_{j=0}^{m} \omega_{j}(1)
\end{aligned}
$$

is fulfilled, where $A$ is a positive constant. Since the functions $h(t, 2 m+1+S), \omega_{0}\left(A t^{2}\right)$, and $\omega_{2 j+1}(A t(T-t))(0 \leq j \leq m-1)$ belong to the set $L^{1}(J)$ by assumption $\left(H_{2}\right)$, in order to prove that $\left\{f_{n}\left(t, x_{n}(t), \ldots, x_{n}^{(2 m)}(t)\right)\right\}$ is uniformly integrable on $J$, it suffices to show that the sequences

$$
\left\{\omega_{2 m}\left(A\left|t-\xi_{m-1, n}\right|\right)\right\}, \quad\left\{\omega_{2 j+2}\left(A\left(t-\xi_{j, n}\right)^{2}\right)\right\}, \quad 0 \leq j \leq m-2
$$

are uniformly integrable on $J$. Due to $\int_{0}^{1} \omega_{2 m}(s) \mathrm{d} s<\infty$ and $\int_{0}^{1} \omega_{2 j}\left(s^{2}\right) \mathrm{d} s<\infty$ for $1 \leq j \leq m-1$ by $\left(H_{2}\right)$, this fact follows from [13, Criterion 11.10 (with $b=A$ and $\left.\left.r=1,2\right)\right]$.

\section{The Main Result}

The following theorem is the existence result for the singular problem (1.1), (1.2).

Theorem 4.1. Let $\left(H_{1}\right)$ and $\left(H_{2}\right)$ hold. Then, problem (1.1), (1.2) has a positive solution $x \in A C^{2 m}(J)$ and

$$
x(t)>0 \quad \text { for } t \in(0, T], \quad(-1)^{j} x^{(2 j+1)}(t)>0 \quad \text { for } t \in(0, T), 0 \leq j \leq m-1 .
$$

Proof. Lemma 3.3 guarantees that problem (2.8), (1.2) has a solution $x_{n}$. Consider the sequence $\left\{x_{n}\right\}$. By Lemma 3.4, $\left\{x_{n}\right\}$ is bounded in $C^{2 m}(J)$,

$$
(-1)^{j} x_{n}^{(2 j+1)}(t)>0 \text { for } t \in(0, T), 0 \leq j \leq m-1,
$$

and $x_{n}$ fulfils estimate (3.29), where $A$ is a positive constant and $\xi_{j, n} \in(0, T)$. Furthermore, the sequence $\left\{f_{n}\left(t, x_{n}(t), \ldots, x_{n}^{(2 m)}(t)\right)\right\}$ is uniformly integrable on $J$ by Lemma 3.5, and therefore, we deduce from the equality $(-1)^{m} x_{n}^{(2 m+1)}(t)=f_{n}\left(t, x_{n}(t), \ldots, x_{n}^{(2 m)}(t)\right)$ for a.e. $t \in J$ that $\left\{x_{n}^{(2 m)}\right\}$ is equicontinuous on $J$. Now, by the Arzelà-Ascoli theorem and the BolzanoWeierstrass theorem, we may assume without loss of generality that $\left\{x_{n}\right\}$ is convergent in $C^{2 m}(J)$ and $\left\{\xi_{j, n}\right\}$ is convergent in $\mathbb{R}$ for $0 \leq j \leq m-1$. Let $\lim _{n \rightarrow \infty} x_{n}=x$ and $\lim _{n \rightarrow \infty} \xi_{j, n}=\xi_{j}$ 
$(0 \leq j \leq m-1)$. Then $x \in C^{2 m}(J)$ and $x$ satisfies the boundary conditions (1.2). Letting $n \rightarrow \infty$ in (3.29) and (4.2), we get (for $t \in J$ )

$$
\begin{gathered}
\left|x^{(2 m)}(t)\right| \geq A\left|t-\xi_{m-1}\right|, \quad\left|x^{(2 j+2)}(t)\right| \geq A\left(t-\xi_{j}\right)^{2} \quad \text { if } 0 \leq j \leq m-2 \\
(-1)^{j} x^{(2 j+1)}(t) \geq A t(T-t) \quad \text { if } 0 \leq j \leq m-1, x(t) \geq A t^{2} .
\end{gathered}
$$

Keeping in mind the definition of $f_{n}$, we conclude from (4.3) that

$$
\lim _{n \rightarrow \infty} f_{n}\left(t, x_{n}(t), \ldots, x_{n}^{(2 m)}(t)\right)=f\left(t, x(t), \ldots, x^{(2 m)}(t)\right) \quad \text { for a.e. } t \in J
$$

Then, by the Vitali theorem, $f\left(t, x(t), \ldots, x^{(2 m)}(t)\right) \in L^{1}(J)$ and

$$
\lim _{n \rightarrow \infty} \int_{0}^{t} f_{n}\left(s, x_{n}(s), \ldots, x_{n}^{(2 m)}(s)\right) \mathrm{d} s=\int_{0}^{t} f\left(s, x(s), \ldots, x^{(2 m)}(s)\right) \mathrm{d} s \quad \text { for } t \in J
$$

Letting $n \rightarrow \infty$ in the equality

$$
x_{n}^{(2 m)}(t)=x_{n}^{(2 m)}(0)+\int_{0}^{t} f_{n}\left(s, x_{n}(s), \ldots, x_{n}^{(2 m)}(s)\right) \mathrm{d} s
$$

we get

$$
x^{(2 m)}(t)=x^{(2 m)}(0)+\int_{0}^{t} f\left(s, x(s), \ldots, x^{(2 m)}(s)\right) \mathrm{d} s \quad \text { for } t \in J
$$

As a result, $x \in A C^{2 m}(J)$ and $x$ is a solution of (1.1). Consequently, $x$ is a positive solution of problem (1.1), (1.2) and inequality (4.1) follows from (4.3).

Example 4.2. Consider problem (1.1), (1.2) with

$$
f\left(t, x_{0}, \ldots, x_{2 m}\right)=p(t)+\sum_{k=0}^{2 m}\left(a_{k}(t)\left|x_{k}\right|^{\alpha_{k}}+\frac{b_{k}(t)}{\left|x_{k}\right|^{\beta_{k}}}\right)
$$

on $J \times \Phi$, where $p, a_{k} \in L^{1}(J), b_{k} \in L^{\infty}(J)$ (that is, $b_{k}$ is essentially bounded and measurable on $J$ ) are nonnegative, $p(t) \geq a>0$ for a.e. $t \in J$. If $\alpha_{k} \in[0,1)$ for $0 \leq k \leq 2 m$ and $\beta_{2 k} \in[0,1 / 2)$, $\beta_{2 m}, \beta_{2 k+1} \in[0,1)$ for $0 \leq k \leq m-1$, then, by Theorem 4.1 , the problem has a positive solution $x \in A C^{2 m}(J)$ satisfying inequality (4.1).

\section{Acknowledgment}

This work was supported by the Council of Czech Government MSM no. 6198959214. 


\section{References}

[1] R. P. Agarwal, S. Pinelas, and P. J. Y. Wong, "Complementary Lidstone interpolation and boundary value problems," Journal of Inequalities and Applications, vol. 2009, Article ID 624631, 30 pages, 2009.

[2] R. P. Agarwal, Boundary Value Problems for Higher Order Differential Equations, World Scientific, Teaneck, NJ, USA, 1986.

[3] R. P. Agarwal and P. J. Y. Wong, "Lidstone polynomials and boundary value problems," Computers $\mathcal{E}$ Mathematics with Applications, vol. 17, no. 10, pp. 1397-1421, 1989.

[4] J. M. Davis, J. Henderson, and P. J. Y. Wong, "General Lidstone problems: multiplicity and symmetry of solutions," Journal of Mathematical Analysis and Applications, vol. 251, no. 2, pp. 527-548, 2000.

[5] Y. Guo and Y. Gao, "The method of upper and lower solutions for a Lidstone boundary value problem," Czechoslovak Mathematical Journal, vol. 55(130), no. 3, pp. 639-652, 2005.

[6] Y. Ma, "Existence of positive solutions of Lidstone boundary value problems," Journal of Mathematical Analysis and Applications, vol. 314, no. 1, pp. 97-108, 2006.

[7] P. J. Y. Wong and R. P. Agarwal, "Results and estimates on multiple solutions of Lidstone boundary value problems," Acta Mathematica Hungarica, vol. 86, no. 1-2, pp. 137-168, 2000.

[8] Y.-R. Yang and S. S. Cheng, "Positive solutions of a Lidstone boundary value problem with variable coefficient function," Journal of Applied Mathematics and Computing, vol. 27, no. 1-2, pp. 411-419, 2008.

[9] B. Zhang and X. Liu, "Existence of multiple symmetric positive solutions of higher order Lidstone problems," Journal of Mathematical Analysis and Applications, vol. 284, no. 2, pp. 672-689, 2003.

[10] R. P. Agarwal, D. O'Regan, I. Rachůnková, and S. Staněk, "Two-point higher-order BVPs with singularities in phase variables," Computers $\mathcal{E}$ Mathematics with Applications, vol. 46, no. 12, pp. 17991826, 2003.

[11] R. P. Agarwal, D. O’Regan, and S. Staněk, "Singular Lidstone boundary value problem with given maximal values for solutions," Nonlinear Analysis: Theory, Methods E Applications, vol. 55, no. 7-8, pp. 859-881, 2003.

[12] I. Rachůnková, S. Staněk, and M. Tvrdý, "Singularities and Laplacians in boundary value problems for nonlinear ordinary differential equations," in Handbook of Differential Equations: Ordinary Differential Equations. Vol. III, A. Cañada, P. Drábek, and A. Fonda, Eds., Handb. Differ. Equ., pp. 607-722, Elsevier/North-Holland, Amsterdam, The Netherlands, 2006.

[13] I. Rachůnková, S. Staněk, and M. Tvrdý, Solvability of Nonlinear Singular Problems for Ordinary Differential Equations, vol. 5 of Contemporary Mathematics and Its Applications, Hindawi Publishing Corporation, New York, NY, USA, 2008.

[14] Z. Wei, "Existence of positive solutions for $n$ th-order singular sublinear boundary value problems," Journal of Mathematical Analysis and Applications, vol. 306, no. 2, pp. 619-636, 2005.

[15] Z. Zhao, "On the existence of positive solutions for $n$-order singular boundary value problems," Nonlinear Analysis: Theory, Methods \& Applications, vol. 64, no. 11, pp. 2553-2561, 2006.

[16] R. G. Bartle, A Modern Theory of Integration, vol. 32 of Graduate Studies in Mathematics, American Mathematical Society, Providence, RI, USA, 2001.

[17] I. P. Natanson, Theorie der Funktionen einer reellen Veränderlichen, Mathematische Lehrbücher und Monographien, Akademie, Berlin, USA, 1969.

[18] D. J. Guo and V. Lakshmikantham, Nonlinear Problems in Abstract Cones, vol. 5 of Notes and Reports in Mathematics in Science and Engineering, Academic Press, Boston, Mass, USA, 1988.

[19] M. A. Krasnosel'skii, Positive Solutions of Operator Equations, P. Noordhoff, Groningen, The Netherlands, 1964

[20] R. P. Agarwal and P. J. Y. Wong, Error Inequalities in Polynomial Interpolation and Their Applications, vol. 262 of Mathematics and Its Applications, Kluwer Academic Publishers, Dordrecht, The Netherlands, 1993. 